DOI: $10.35643 /$ Info.26.1.12

\author{
Dossier temático: Ética de la Información
}

\title{
Los códigos éticos de los profesionales bibliotecólogos del Uruguay: análisis desde el Sistema de Valoración
}

\author{
The Ethical Codes of Professional Librarians in Uruguay: Analysis from the \\ Appraisal System
}

Os códigos éticos dos bibliotecários profissionais no Uruguai: análise do Sistema de Avaliação

\author{
Maximiliano Rodríguez-Fleitas ${ }^{\mathrm{a}}$
}

a Facultad de Información y Comunicación, Universidad de la República, Uruguay. ORCID: 0000-0002-5881-845X. Correo electrónico: maximiliano.rodriguez@,fic.edu.uy

\section{Resumen}

Surge de la literatura consultada que los profesionales de la información deben afrontar, en mayor y menor medida, diferentes conflictos éticos relacionados con su labor profesional y, por lo tanto, con la producción, recopilación, acceso y difusión de la información. Los códigos deontológicos buscan de alguna forma plasmar los principios y valores que permiten superar estos conflictos y establecer, en cierta medida, un marco normativo para la actividad profesional. En tal sentido, se hace necesario, evaluar el discurso que se expresa a partir del código de ética aprobado por la Asociación de Bibliotecólogos del Uruguay en el año 2000, a los efectos de conocer cómo se representan los bibliotecólogos uruguayos y qué valores son los que se asocian con esta comunidad. Se utilizan herramientas propias del análisis del discurso, específicamente se realiza un análisis a partir del sistema de valoración de Martin y White (2005). El análisis demuestra una predominancia de los significados de juicio intensificados a partir de verbos modales de obligación.

Palabras clave: ÉTICA PROFESIONAL; CÓDIGOS DE ÉTICA; ÉTICA BIBLIOTECOLÓGICA; TEORIA DE LA VALORACION.

\begin{abstract}
It emerges from the literature consulted that information's professionals must face, somehow, different ethical conflicts related to their professional work and, therefore, with the production, collection, access and dissemination of information. Deontological codes seek in some way to capture the principles and values that make it possible to overcome these conflicts and establish a regulatory
\end{abstract}


framework for professional activity. To that effect, it is necessary to evaluate the discourse that is expressed from the code of ethics approved by the Uruguayan Association of Librarians in 2000, in order to know how Uruguayan librarians are represented and what values are those that they associate with this community. Discourse analysis tools are used, specifically the appraisal system of Martin and White (2005). The analysis shows a predominance of heightened judgment meanings from modal verbs of obligation.

Keywords: PROFESSIONAL ETHICS; CODES OF ETHICS; LIBRARY ETHICS; APPRAISAL THEORY.

\section{Resumo}

Decorre da literatura consultada que os profissionais da informação devem enfrentar, em maior ou menor medida, diferentes conflitos éticos relacionados com o seu trabalho profissional e, portanto, com a produção, recolha, acesso e divulgação da informação. Os códigos deontológicos procuram, de alguma forma, captar os princípios e valores que possibilitam a superação desses conflitos e estabelecer, em certa medida, um marco regulatório da atividade profissional. Nesse sentido, é necessário avaliar o discurso que se expressa a partir do código de ética aprovado pela Associação Uruguaia de Bibliotecários em 2000, para saber como os bibliotecários uruguaios estão representados e quais valores estão associados a esta comunidade. São utilizadas ferramentas de análise de discurso, especificamente uma análise é realizada com base no sistema de avaliação de Martin e White (2005). A análise mostra uma predominância de significados elevados de julgamento de verbos modais de obrigação.

Palavras-chave: ÉTICA PROFISSIONAL; CÓDIGOS DE ÉTICA; ÉTICA DA BIBLIOTECA; TEORIA DA AVALIAÇÃO.

Fecha de recibido: $15 / 01 / 2021$

Fecha de aceptado: 02/05/2021

\section{Evolución de los dilemas y principios éticos de la profesión bibliotecológica}

La breve síntesis de la evolución de los dilemas y principios éticos que se presenta a continuación, sirve como preámbulo al abordaje de los códigos éticos de la profesión bibliotecológica a partir de metodologías del análisis del discurso. En particular, utilizaremos como herramientas de análisis el sistema de valoración expresado en la Teoría de la Valoración de Martin y White (2005). Como menciona Pascual (2017), esta teoría se concentra en el análisis cualitativo y brinda al analista la posibilidad de analizar de forma sistémica, no solo lo 
señalado lingüísticamente, sino también lo evocado a través del lenguaje. Por lo tanto, comenzar a descifrar el contexto en el cual se sitúa nuestro corpus, que desarrollaremos más adelante, se hace fundamental.

En particular, los dilemas éticos se consideran situaciones en las que existen obligaciones éticas en conflicto, y hacer una cosa correcta tiene el potencial de causar daño de otra manera (Ferguson et al, 2016). Los profesionales de la información deben afrontar, en mayor y menor medida, diferentes conflictos éticos relacionados con su labor profesional.

Por ejemplo, en la década de los 90, Capurro entrevistado por Gutiérrez (2009), puntualiza que los dilemas relacionados con el acceso a la red de Internet comienzan a acaparar el debate ético en el campo de la información. Como menciona Juznic (2001), este nuevo escenario obliga a replantearse los dilemas que pueden surgir de la incorporación de las nuevas tecnologías y esto debería impulsar un debate serio que supere una visión profesional centrada en el dominio de la imprenta.

Por lo tanto, autores como Capurro (2005 y 2009) y Froehlich (1999a), consideran que Internet y la potencialidad de la interacción multimedia que ofrecen las nuevas tecnologías de la información, es motivo de por sí de muchas preocupaciones generales, por ejemplo, la capacitación en el uso de estas tecnologías, su apropiación social y cultural, aspectos relacionados con la privacidad, la confidencialidad, la ciberseguridad, la libertad intelectual y la libertad de expresión, el derecho de acceso a la información gubernamental, conflictos con respecto a los derechos de autor y la creación artística, los potenciales riesgos del acceso universal y no mediado a la información, el carácter problemático de las herramientas de la red, entre otros.

Por su parte, Hauptman en conversación con Buchanan (2008), comparte dichas problemáticas generales, aunque considera que mientras las tecnologías han cambiado, los dilemas que enfrentan los profesionales de la información no son significativamente diferentes, pero si se han agravado.

Existen valores y principios éticos a los cuales los profesionales pueden apelar para clarificar y resolver estos dilemas éticos. Aunque, como señalan algunos 
autores, la pluralidad en la tarea de los profesionales de la información, el contexto y los usuarios, plantean una diversidad de cuestiones éticas.

Para Froehlich (1999b), podríamos reconocer algunos valores y principios fundamentales, los mismos se relacionan con el respeto a la autonomía propia y ajena que formulara Kant en su imperativo categórico: trata a los seres humanos como fines y nunca simplemente como medios. De este principio se desprenden valores como la libertad, la autodeterminación, la protección frente a daños de terceros, la igualdad de oportunidades, la privacidad, el promover al bienestar de los otros y reconocer su trabajo.

Otros de los principios que señala Froehlich (1999b) son la búsqueda de la justicia o la imparcialidad a partir del respeto por los demás, al que hacíamos referencia anteriormente, la búsqueda de la armonía social a partir de beneficiar a la mayor cantidad de usuarios posibles, el actuar previendo el mínimo prejuicio final, fortalecer la confianza en la organización profesional y los usuarios a partir de los principios y la deliberación ética de los dilemas.

Para autores como Capurro (1985), específicamente y relacionado con la diseminación de la información surgen tres principios: el principio de la accesibilidad, el principio de la confidencialidad y el principio de compleción.

El primero garantiza que la información esté disponible para todo el que la necesite, pero teniendo en cuenta las diferentes restricciones políticas, económicas, entre otras; este principio adquiere un valor significativo. Como señala Capurro (1985), pueden existir conflictos entre naciones 'ricas en información' y 'pobres en información', conflictos de información entre naciones desarrolladas, cuestiones de monopolios y controles democráticos, etc.

El principio de confidencialidad afecta a la responsabilidad de los divulgadores de la información, tanto con su propia información, como con los datos personales de productores y usuarios.

Por último, el principio de compleción garantiza la integridad y confiabilidad de la información gestionada, conservada y diseminada. Por ejemplo, en el caso de un productor de base de datos, este principio apunta a la posibilidad de 
discriminación de información o de selectividad sesgada, a través de la cual los usuarios podrían ser engañados. (Capurro, 1985)

Los códigos deontológicos buscan convertirse en la expresión escrita de este conjunto de deberes y principios que un colectivo de trabajadores, de una determinada profesión, ha ido descubriendo a través del ejercicio de dicha actividad y señala los límites medianamente seguros para obrar bien. Es, pues, un conjunto de buenas prácticas destinado a alcanzar el conjunto de bienes que la profesión pretende en los usuarios (Pantoja Vargas, 2011).

En la misma línea, Frankel (1989) señala que la concesión de poder y privilegio de la sociedad a las profesiones se basa en su buena voluntad y capacidad de contribuir al bienestar social y a conducir sus asuntos de manera consistente con valores sociales más amplios. Los códigos buscan encarnar dichos valores y explicitan la conciencia colectiva de una profesión y su dimensión moral.

El primer código de ética de la profesión bibliotecológica fue formulado y ratificado por la American Library Association en el año 1938, posteriormente sufrió varias revisiones, y todavía es activamente utilizado y considerado. Sus antecedentes están ligados a la profesionalización de la tarea de los bibliotecarios norteamericanos y a la formulación de las primeras declaraciones éticas de la profesión elaboradas por Plummer en 1903 y Bolton en 1909. (Hansson, 2016)

Justamente la declaración de Bolton (1922) se convertiría en la versión preliminar del código aprobado en 1938. La misma consta de 30 "cánones", o párrafos, que definen áreas dentro de la práctica profesional donde las cuestiones éticas pueden entrar en tensión.

En el preámbulo del mencionado código se reconoce que al influir o controlar significativamente la selección, organización, preservación y difusión de información, en un sistema político basado en una ciudadanía informada, los bibliotecarios se identifican como miembros de una profesión explícitamente comprometida con la libertad intelectual y la libertad de acceso a la información. Con la obligación especial de garantizar el libre flujo de información e ideas a las generaciones presentes y futuras. (American Library Association, 2008) 
En los años venideros diferentes asociaciones profesionales nacionales irían adoptando sus propios códigos deontológicos. Sin embargo, durante 2010 y 2012, un grupo de trabajo del Comité de Libre Acceso a la Información y Libertad de Expresión (FAIFE por sus siglas en inglés) de la Federación Internacional de Bibliotecarios Asociados (IFLA por sus siglas en inglés), redactó y revisó ampliamente un código de ética internacional para los bibliotecarios y otros trabajadores de la información. El mismo contó con el apoyo de Globethics, una red global de profesores e instituciones de educación superior que tiene la visión de integrar la ética en sus actividades profesionales.

El código aprobado en el año 2012 desarrolla los valores fundamentales promovidos por el artículo 19 de la Declaración Universal de los Derechos Humanos de las Naciones Unidas (1948):

Todo individuo tiene derecho a la libertad de opinión y de expresión; este derecho incluye el de no ser molestado a causa de sus opiniones, el de investigar y recibir informaciones y opiniones, y el de difundirlas, sin limitación de fronteras, por cualquier medio de expresión.

En tal sentido, el código establece como principios fundamentales en la labor profesional de los bibliotecólogos el garantizar el acceso a la información de forma universal, la lucha por preservar y promover espacios y servicios que garanticen la libertad de expresión y la lucha contra la censura, promover la responsabilidad social, el respeto por la privacidad, confidencialidad y la promoción de la transparencia; el apoyo al acceso abierto y la defensa de excepciones y limitaciones en materia de derecho de autor, la neutralidad, integridad personal y el desarrollo de habilidades profesionales en pro de la excelencia; el trato justo y respetuoso con otros colegas (International Federation of Library Associations and Institutions, 2012).

\section{Breve historia de la Asociación de Bibliotecólogos del Uruguay y creación de sus códigos de ética}

En Uruguay el origen y desarrollo de la asociación de profesionales bibliotecólogos y su código de ética, está íntimamente relacionado con el desarrollo técnico de la profesión. 
Si bien la preocupación por contar con personal calificado en la dirección y gestión de las bibliotecas uruguayas se remonta a finales del siglo XIX y principios del siglo XX (Carrazone, 2018). Recién en la década del 40, tras el impulso y trabajo del ingeniero Federico E. Capurro y el bibliotecario estadounidense Arthur E. Gropp, se establecen los primeros cursos y se funda la Escuela de Bibliotecnia (Capurro, 1950).

En la inauguración de los cursos en la Asociación de Ingenieros, el 23 de marzo de 1943, Arthur E. Gropp menciona:

Pero una Escuela no puede llevar a cabo el ideal de la organización de bibliotecas si no cuenta con la ayuda y cooperación de los bibliotecarios. En Norte América un grupo de bibliotecarios creó para este efecto la Asociación Americana de Bibliotecas, que hoy día cuenta con un número de más de 15.000 socios. Con esta sugerencia dejo la palabra, y deseo que la próxima acción en favor del desarrollo de la profesión bibliotecaria sea la creación de una Asociación de Bibliotecarios del Uruguay. (Santestevan, R., 2003).

Como señala Santestevan (2003), los estatutos para la creación de la Asociación de Bibliotecarios Diplomados del Uruguay fueron aprobados el 12 de mayo de 1945 por una asamblea de bibliotecarios y el 8 de agosto del mismo año por el Ministerio de Instrucción Pública y Previsión Social.

Esta asociación promueve varios encuentros e instancias de intercambio profesionales hasta la década del 70, años en que el recorte de libertades institucionales dificulta mantener activa la participación de los agremiados en la asociación. Finalmente, luego de varios intentos de reforma de estatuto de la antigua asociación, en el año 1978 se establece la vigente Asociación de Bibliotecólogos del Uruguay.

Recién en el año 1981 se adopta el primer código de ética de la nueva asociación, el mismo es posteriormente revisado por una Comisión a partir de la presentación de un documento base puesto a consideración en el "Seminario de evaluación del ejercicio profesional de la Bibliotecología en el Uruguay 1943-1982” celebrado el año 1983 en Montevideo (Cano et al, 1983).

En dicho documento se establecen ideales o principios éticos para la profesión y una propuesta de pautas concretas y prácticas de actuación profesional con la finalidad de promover el desarrollo de un nuevo y actualizado código de ética. Se 
hace especial hincapié en la necesidad de que los profesionales asuman su condición de bibliotecólogos de forma íntegra y eficiente, promuevan el respeto para su labor y sus colegas. Por otro lado, se promueve la independencia intelectual del profesional a partir del principio de imparcialidad a los efectos de garantizar el acceso ecuánime de los usuarios a la información. (Cano et al, 1983)

Finalmente, en el año 2000 la asamblea general de la Asociación de Bibliotecólogos del Uruguay (2000), aprueba el vigente código de ética que analizaremos a continuación. Cabe mencionar que la carencia de estudios a nivel local en estos veinte años, impide fundamentar la pertinencia y relevancia de los códigos deontológicos nacionales de las profesiones de la información. Esto hace suponer que un análisis científico debería aportar nuevos insumos al debate de sus particularidades, pertinencia y actualidad.

\section{Teoría de la Valoración}

El presente trabajo aborda el estudio del código ético de los bibliotecólogos uruguayos a partir de herramientas y categorías de análisis desarrolladas por Martin y White (2005) a partir de la teoría de la valoración.

Como expresa White (2000), la Teoría de la Valoración se ocupa de los recursos lingüísticos por medio de los cuales los textos/hablantes llegan a expresar, negociar y naturalizar determinadas posiciones intersubjetivas y en última instancia, ideológicas. Dentro de esta amplia área de interés, la teoría se ocupa particularmente del lenguaje (la expresión lingüística) de la valoración, la actitud y la emoción, y del conjunto de recursos que explícitamente posicionan de manera interpersonal las propuestas y proposiciones textuales. En tal sentido, se identifican tres grandes subsistemas: el actitudinal, el de graduación y el de compromiso.

La actitud construye evaluaciones a partir de tres categorías: el afecto, que evalúa los recursos para expresar los sentimientos; el juicio, para evaluar las actitudes hacia la conducta y el carácter y, por último, la apreciación, que permite evaluar el valor de los objetos, productos materiales y no materiales con principios estéticos u otros valores sociales (Martin y White, 2005). 
La graduación, por su parte, se relaciona con el aumento y la reducción de la escala. Es entendida como una propiedad general de los diferentes subsistemas e interpreta grados mayores o menores de positividad o negatividad. Este subsistema se divide en dos partes, por un lado, tenemos la fuerza, que cubre evaluaciones relacionadas con el grado de intensidad y cantidad; por el otro lado el foco, que cubre la especificación o suavización de los bordes entre categorías en el contexto de los elementos no graduables (Martin y White, 2005).

Por último, el tercer recurso evaluativo es el compromiso, el mismo refiere a los recursos que permiten posicionar la voz del hablante/autor en relación con las diversas proposiciones y comunidades a través del texto; los significados por medio de los cuales los hablantes reconocen o ignoran la diversidad de puntos de vista que sus emisiones ponen en juego y por medio de las cuales negocian un espacio interpersonal para sus propias posiciones dentro de esa diversidad (White, 2000).

\section{Corpus y metodología}

Se selecciona como corpus de este trabajo la última versión del código de ética de la Asociación de Bibliotecólogos del Uruguay. Como vimos anteriormente, los códigos éticos funcionan como herramientas que buscan expresar los valores de un cuerpo profesional y establecen un marco de referencia para la actuación de una comunidad profesional.

En tal sentido, se hace fundamental evaluar el discurso que se expresa a partir de este documento normativo, a los efectos de reconocer cómo se representan los bibliotecólogos uruguayos y qué valores son los que se asocian con esta comunidad. No existen antecedentes de estudio o evaluación de estas herramientas, por lo que los resultados de este trabajo seguramente aporten nuevas líneas de profundización.

Como se expresa en el apartado de objetivos y finalidades del código de ética de la ABU (2000):

El siguiente Código de Ética está orientado a establecer un marco normativo para las actividades que se desarrollen por la Asociación de Bibliotecólogos del Uruguay, a efectos de lograr los fines primordiales, definidos como objetivos, conforme a los Art. 1 y 2 de los Estatutos. 
Con la finalidad de cumplir con estos objetivos de investigación nos centraremos en los recursos de valoración actitudinales, específicamente en el subsistema de juicio. Este carácter normativo nos condiciona a centrarnos en los juicios de sanción social, como menciona Martin (apud Pascual 2017), los mismos tienen que ver con la veracidad y la corrección. Por lo que es de esperar que dentro de los resultados se perciba una predominancia de los enunciados de corrección.

Por otro lado, debemos tener en cuenta que como menciona Pascual (2017), los juicios de sanción social son recursos valorativos que construyen la posición del autor con respecto a la ética y la conducta de los actores sociales.

Se analiza además la entidad evaluadora, la entidad evaluada y se incorporan herramientas del subsistema de graduación de los enunciados para reconocer categorías de escala semántica relacionadas con los valores expresados en el código analizado.

Por último, cabe señalar, que el código de ética de los bibliotecólogos uruguayos se divide en 5 capítulos que integran un total de 27 artículos, el primero define los objetivos y finalidades del código. Los restantes capítulos se dividen en artículos centrados con la gestión profesional, el ejercicio liberal, la función gremial y, por último, la cooperación y el desarrollo.

\section{Resultados}

En total se analizaron 27 instancias evaluativas, correspondientes a los artículos que componen el código de ética. Como era de esperar, en su mayoría, permiten ser categorizadas dentro del subsistema de juicio.

Como menciona Pascual (2017), los valores de juicio son los que solidariamente construyen una cultura a lo largo de generaciones. Existe una preocupación por delimitar el rol profesional de los bibliotecólogos en el Uruguay a partir de la construcción de un ideal de buenas prácticas y la construcción de principios y valores comunes para todos estos profesionales.

Se reconoce en primera instancia la responsabilidad moral y judicial de los profesionales. A modo de ejemplo, se analiza una de las primeras instancias de nuestro corpus: 
Art. 2.2: El bibliotecólogo (entidad evaluada) es responsable moral y jurídicamente de sus actos profesionales (juicio de sanción social).

Aquí identificamos la entidad evaluada predominante en el texto, el bibliotecólogo, el profesional que es evaluado en su tarea profesional por su misma asociación profesional (entidad evaluada), productora del discurso integrado al código de ética analizado. El valor de responsabilidad es recurrente en el artículo centrado en la gestión profesional, en el que además se juzga la responsabilidad referida al cuidado de las colecciones, al respeto de las normativas en materia de derecho de autor, a la estricta observancia del derecho a la información, entre otros.

Todos estos valores podrían ubicarse dentro del subsistema de adecuación (¿qué tan ético y responsable es?), valor predominante dentro de las cláusulas de sanción social identificadas en el texto:

Art. 2.3: El bibliotecólogo (entidad evaluada) es el responsable del cuidado, de la custodia y de la conservación de las colecciones a su cargo (juicio de sanción social/adecuación).

Solo identificamos una cláusula en la subcategoría de veracidad (¿qué tan honesta y transparente es la persona?) (White, 2000), el artículo 2.7:

Art. 2.7: En su función de intermediación, el bibliotecólogo (entidad evaluada) debe tratar y transmitir la información en forma confiable, válida y sistemática posible (juicio de veracidad), preservando su autonomía técnica e independencia intelectual.

Con relación a las escalas de graduación, identificamos en las escalas de fuerza que estás pueden operar sobre modalidades verbales de obligación (Martin y White, 2005). Al analizar las escalas de graduación identificamos una predominancia del verbo modal "debe", en total son 17 instancias (63\% del total) que lo integran. El mismo reafirma el carácter alto de la obligatoriedad en las instancias evaluadas: 
Art. 2.5: Debe reconocer y respetar los derechos de propiedad intelectual y normas atinentes.

Art. 3.1: En el ejercicio liberal, debe mostrar permanentemente idoneidad, asegurando el respeto por la jerarquía intelectual y por la propia naturaleza profesional de la función.

Art. 5.2: Considerando el beneficio de la interdisciplinariedad, el bibliotecólogo debe estar abierto a aceptar y promover programas comunes con otras profesiones.

Como se aprecia, identificamos este término de intensidad a la hora de evaluar la idoneidad de los profesionales, el estricto cumplimiento de las normas legales relacionadas con la labor, el apego a la normativa que aseguren transparencia en la administración de las instituciones gremiales, la apertura a la interdisciplinariedad, la promoción de la investigación científica en su campo de conocimiento, la actualización, etc.

En dos instancias podemos ver también como varía el foco con relación a la entidad evaluada:

Art. 4.1: Como miembro de un cuerpo profesional, el bibliotecólogo (entidad evaluada), en la medida de sus posibilidades (opción), tiene la responsabilidad de participar en el desarrollo y mantenimiento de la Institución que lo agremia.

En ese mismo capítulo, dedicado a la función gremial, posteriormente se utilizan una escala de foco mucho más precisa a la anterior, ya que se incluye a todos los bibliotecólogos agremiados, sin excepciones, en el cumplimiento de las disposiciones: 
Art. 4.3: Las disposiciones de este Capítulo son obligatorias para todos los bibliotecólogos agremiados a la ABU. Las infracciones a estas disposiciones se elevarán a la jurisdicción disciplinaria correspondiente.

Cabe mencionar que, como veíamos anteriormente, este capítulo es el que integra diversas herramientas de graduación, lo cual podría interpretarse como una variación del posicionamiento en abordajes relacionados con la agremiación como núcleo para los profesionales de esta disciplina.

\section{Conclusiones y últimas reflexiones}

Este primer y breve abordaje del código de ética de los bibliotecólogos uruguayos, desde el enfoque de la teoría de la valoración, permite identificar enunciados que aportan a la construcción del rol profesional bibliotecólogo desde un discurso concreto y estructurado. A partir de los resultados se identifican formas específicas del uso del lenguaje en este tipo de documentos, en el que se expresa el contexto ideológico y social en el que están insertos.

La predominancia de instancias de juicio moduladas por un fuerte apego a ciertas obligaciones, trazan cierta adherencia a ciertos valores percibidos como positivos dentro de la comunidad profesional evaluada (los bibliotecólogos) y quién evalúa (su asociación profesional).

Podríamos afirmar, además, que la prevalencia del verbo modal "debe" se alinea con la tradición deontológica de procedencia kantiana. Como mencionábamos anteriormente, al hacer referencia a los valores y principios identificados por Froehlich (1999b) a partir del respeto a la autonomía propia y ajena que formulara Kant en su imperativo categórico: trata a los seres humanos como fines y nunca simplemente como medios. Permite desprenden valores presentes en este código profesional, como son la libertad, la autodeterminación, la protección frente a daños de terceros, la igualdad de oportunidades, la privacidad, la promoción al bienestar de los otros y el reconocimiento al trabajo de colegas y compañeros.

Además de los valores relacionados con la diseminación y tratamiento de la información, desarrollados también anteriormente, como son la accesibilidad, confidencialidad y compleción. 
Todos estos principios éticos se encuentran alineados en mayor y menor medida con la Declaración de Derechos Humanos de las Naciones Unidas, principalmente el artículo 19, que declara como derecho la libertad de expresión y el acceso a la información.

Si bien el código analizado ya supera los 20 años desde su aprobación, como podemos observar, muchas de las preocupaciones y obligaciones expresadas para los profesionales se entienden como vigentes o en proceso de agravarse.

En tal caso, sería importante profundizar el estudio de estas herramientas y analizar desde diversas miradas el proceso social e histórico que permitió el desarrollo de la profesión, su asociación y su eventual código de ética. En el entendido de que el contexto en el que se utiliza la información no solo está mediado por los profesionales, sino también por los usuarios y las instituciones.

Como señalan y profundizan varios autores, entre ellos Capurro (1985) y Ladd (1985), la cuestión clave para una sociedad profesional en materia de ética no es establecer solamente un código, sino promover la discusión sobre las cuestiones éticas relacionadas con sus actividades. Por lo tanto, esperemos que a partir de este pequeño aporte puedan motivarse nuevos espacios de estudio y discusión.

\section{Bibliografía}

American Library Association. (2008). Professional ethics. Recuperado de http://www.ala.org/tools/ethics

Asociación de Bibliotecólogos del Uruguay. (2000). Código de ética. Recuperado de http://www.abu.net.uy/la-asociacion/codigo-de-etica/

Bolton, C. K. (1922). The Ethics of Librarianship: A Proposal for a Revised Code. The Annals of the American Academy of Political and Social Science, 101, 138-146.

Buchanan, E. A. (2008). On theory, practice, and responsibilities: a conversation with Robert Hauptman. Library \& Information Science Research, 30(4), 250-256.

Capurro, F. E., (1950). Una memoria más: 1898 - 1948. Montevideo. Recuperado de http://capurro.de/capurro_bibliotecnia.html 
Capurro, R. (1985). Moral issues in information science. Journal of Information Science, 11, 113-123.

Capurro, R. (2005). Ética de la información: un intento de ubicación. Códices, 1 2, 87-95. Recuperado de http://www.capurro.de/colombia.htm

Capurro, R., Gutiérrez, D. (2009). Ética Intercultural de la información. En H. Ferreira Gomes, A., Bottentuit, M., Espinheiro de Oliveira, M. O. (Eds.), Ética na sociedade, na área da informação e da atuação profissional. pp. 43-64. Conselho Federal de Biblioteconomia.

Cano, R., Barité, M., Torres, F. (1983). Ética bibliotecológica. Asociación de Bibliotecólogos del Uruguay.

Carrazzone, J. (2018). Ensayo final de pasantía: Proceso de fundación de la Biblioteca del Poder Legislativo en Uruguay. Antecedentes y discusión parlamentaria de la aprobación de la Ley 8417 que unificó a las bibliotecas de la Cámara de Senadores y Diputados (1928-1929). Tutor: Juan Andres Moraes. Montevideo: Universidad de la República, Facultad de Ciencias Sociales.

Ferguson, S., et al (2016). Beyond codes of ethics: how library and information professionals navigate ethical dilemmas in a complex and dynamic information environment. International Journal of Information Management, 36, 543-556.

Frankel, M. (1989). Profesional codes: why, how and with what impact? Journal of Business, 8, 109-115.

Froehlich, T. (1999a). Las preocupaciones éticas de los profesionales de la información acerca de Internet. Educación y biblioteca, 106, 61-69.

Froehlich, T. (1999b). Un marco general para pensar acerca de las cuestiones éticas en bibliotecas y en centros de información. Educación y biblioteca, 99, 46-56.

Hansson, J. (2016). The Documentality of Ethics - Codes of Library Ethics as Support of Professional Practice. Proceedings from the Document Academy, 3, 1. DOI: https://doi.org/10.35492/docam/3/1/8

International Federation of Library Associations and Institutions. (2012). Código de Ética de la IFLA Para Bibliotecarios y Otros Trabajadores de la Información. Recuperado de https://www.ifla.org/node/7237 
Juznic, P., et al (2001). Excuse me, how do I commit suicide? Access to Ethically Disputed Items of Information in Public Libraries. Library Management, $22,1 / 2,75-80$.

Ladd, J. (1985). The quest for a code of professional ethics: an intellectual and moral confusion. En: Deborah G. Johnson y J. W. Snapper (Eds.), Ethical Issues in the Use of Computers. p. 8-13. Belmont: Wadsworth.

Martin, J. R., White, P. R. R. (2005). The Language of Evaluation: Appraisal in English. London/New York: Palgrave/Macmillan.

Naciones Unidas. (1948). Declaración Universal de Derechos Humanos.

Recuperado de

https://www.ohchr.org/EN/UDHR/Documents/UDHR Translations/spn.p $\underline{\mathrm{df}}$

Pantoja Vargas, L. (2011). Deontología y código deontológico del Educador Social, Pedagogía Social. Revista Interuniversitaria, 19, 65-79.

Pascual, M. (2017). La violación de los derechos humanos en la Argentina: dinámica evaluativa de dos décadas de representación en medios. Discurso \& Sociedad, 11, 3, 388-413. Recuperado de https://dialnet.unirioja.es/servlet/articulo?codigo $=6140838$

Santestevan, R. (2003). Sintesis histórica ABU. Recuperado de https://www.abu.net.uy/la-asociacion/historia/

White, P. R. R. (2000). Un recorrido por la teoría de la valoración (Teoría de la valoración). Recuperado de https://www.researchgate.net/publication/255625569 Un recorrido por 1 a teoria de la valoracion

\section{Author contribution}

The entirety of this manuscript was prepared by Maximiliano Rodríguez-Fleitas.

\section{Editor's notes}

The editor responsible for the publication of this article was Rafael Capurro. 